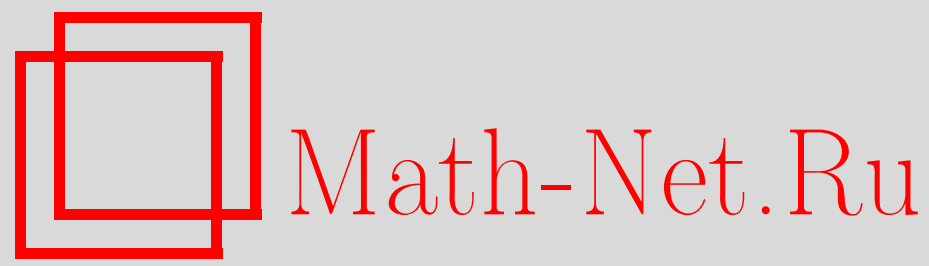

Ю. В. Покорный, В. Л. Прядиев, А. Аль-Обейд, Об осцилляционности спектра краевой задачи на графе, Матем. заметки, 1996, том 60, выпуск $3,468-470$

DOI: https://doi.org/10.4213/mzm1853

Использование Общероссийского математического портала Math$\mathrm{Net.Ru}$ подразумевает, что вы прочитали и согласны с пользовательским соглашением

http://www . mathnet.ru/rus/agreement

Параметры загрузки:

IP : 34.229 .45 .116

26 апреля 2023 г., 14:41:25

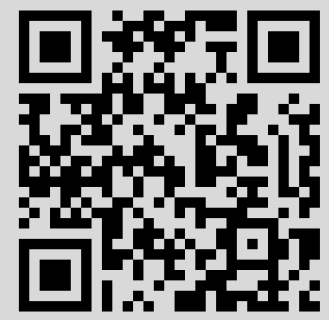




\section{ОБ ОСЦИЛЛЯЦИОННОСТИ СПЕКТРА КРАЕВОЙ ЗАДАЧИ НА ГРАФЕ}

\section{Ю. В. Покорный, В. Л. Прядиев, А. Аль-Обейд}

В работе показывается, что осцилляционные спектральные свойства Штурма для задачи

$$
-\left(p u^{\prime}\right)^{\prime}+q u=\lambda r u
$$

на отрезке при $u(a)=0=u(b)$ допускают перенос на случай существенно неодномерной задачи, когда уравнение (1) задается не на интервале $(a, b)$, а на геометрическом графе (пространственной сети) Г при условиях

$$
\left.u\right|_{\partial \Gamma}=0 \text {, }
$$

где $\partial Г$ - множество граничных (тупиковых) вершин $Г$.

1. Краевые задачи на графах (пространственных сетях) предметом систематического анализа стали лишш с начала 80-х годов. В первых работах [1]-[3] уравнение вида (1) на графе Г интерпретировалось в виде набора (системы) обыкновенных скалярных уравнений, каждое из которых задавалось на одном из ребер $\gamma_{i}$ графа $\Gamma$. При этом условие связи между решениями уравнений на смежных ребрах, примыкающих к одной внутренней вершине, истолковьвались как краевые. В качестве таких условий согласования в каждой внутренней вершине $a$ обычно выступают условия непрерьвности, т.е. $u_{i}(a)=u_{j}(a)(i \neq j)$ (здесь $u_{i}(\cdot)$ - сужение решения на ребро $\gamma_{i}$ ) и условия баланса вида

$$
\sum_{i} \alpha_{i}(a) u_{i}^{\prime}(a+0) \quad\left(\alpha_{i}(a)>0\right),
$$

где $u_{i}^{\prime}(a+0)$ обозначает производную $u_{i}(\cdot)$ вдоль ребра $\gamma_{i}$ в направлении "от $a$ ". Достаточно глубокая аналогия с классическими скалярньми задачами была установлена в результате применения другого подхода [4], [5], при котором уравнение (1) рассматривается в классе скалярнозначных функций, определенных в целом на $Г$ при естественном условии их непрерывности во всех внутренних вершинах (т.е. в целом на $\Gamma$ ); условия (3) переносятся в определение решения. При таком подходе к определению решения уравнение (1) на $\Gamma$ превращается в промежуточный объект между скалярным уравнением на отрезке и уравнением второго порядка на многомерной области, что позволяет считать задачу вида (1) , (2) аналогом задачи Дирихле. Ниже используется этот скалярный взгляд на задачу (1), (2) на граффе; точный смысл используемых понятий см. в [4], [5].

2. Пусть $Г$ - открытый связный геометрический графф (в дальнейшем - просто граф) из $\mathbb{R}^{n},\left\{\gamma_{1}, \ldots, \gamma_{m}\right\}$ - набор всех его ребер (прямолинейных интервалов). Как и в [4], [5], через $\partial Г$ обозначается множество граничных, через $J(\Gamma)$ - множество внутренних для $Г$ вершин (каждая из внутренних вершин принадлежит $\Gamma$, причем к ней примькает не менее двух ребер; каждая граничная вершина графу не принадлежит, т.е. $\partial \Gamma \cap \Gamma=\varnothing)$. Подмножество $\Gamma_{0}$ из $\Gamma$ называется подграфом $\Gamma$, если оно связно и открыто в топологии на $\Gamma$, индуцируемой из $\mathbb{R}^{n}$.

Всюду, далее, мы предполагаем, что Г является деревом, т.е. не содержит циклов (подмножеств, гомеоморфных окружности). Это значит, что при выбрасьвании из Г любой точки $x_{0} \in \Gamma$ оставшееся множество $\Gamma \backslash\left\{x_{0}\right\}$ распадается на две или более

Работа выполнена в рамках программы "Университеты России". 
компоненты связности, каждая из которых является подграфом Г. Ниже предполагается, что функции $q(\cdot), r(\cdot)$ и $p^{\prime}(\cdot)$ равномерно непрерывны на каждом ребре $\gamma_{i}$, причем $p(\cdot)$ и $r(\cdot)$ имеют строго положительные нижние грани.

Для описания основного результата нам потребуется понятие общности положения граффа $\Gamma$ для уравнения (1). Для всякой внутренней вершины $a \in J(\Gamma)$ и любого примькающего к этой вершине ребра $\gamma_{i}$ обозначим через $\Gamma_{i}(a)$ подграф $\Gamma$, являющийся компонентой связности множества $\Gamma \backslash\{a\}$ и содержащий $\gamma_{i}$. Обозначим через $\Lambda_{i}(a)$ спектр задачи

$$
\begin{gathered}
-\left(p u^{\prime}\right)^{\prime}+q u=\lambda r u \quad\left(x \in \Gamma_{i}(a)\right), \\
\left.u\right|_{\partial \Gamma_{i}(a)}=0 .
\end{gathered}
$$

Скажем, что граф Г находится в общем положении для уравнения (1), если для любой внутренней вершины $a$ спектры $\Lambda_{i}(a)$ и $\Lambda_{j}(a)$ при $i \neq j$ не пересекаются.

ТЕорема. Пусть Г находится в общем положении для уравнения (1). Тогда спектр $\Lambda$ задачи (1), (2) состоит из неограниченной последовательности $\lambda_{0}<\lambda_{1}<\lambda_{2}<\cdots$ вешественных простых собственных значений. При этом собственная функиия $u_{k}(\cdot)$, отвечающая собственному значению $\lambda_{k}$, имеет в Г точно $k$ нулей, в каждом из которых она меняет знак.

3. Поясним доказательство теоремы. Вещественность спектра $\Lambda$ задачи (1), (2) следует из отсутствия у $Г$ циклов: индуктивньми рассуждениями несложно показывается, что домножением уравнения (1) на константу на каждом ребре $\gamma_{i}$ можно преобразовать задачу (1), (2) к эквивалентной так, что в условиях (3) окажется $\alpha_{i}(a)=p_{i}(a)$. Последняя задача является самосопряженной в естественном смысле.

Так как замена $\lambda=\mu-\mu_{0}$ при $\mu_{0}>0$ сдвигает спектр вправо, не меняя заявленных в теореме его свойств, то мы можем считать коэффициент $q(\cdot)$ в уравнении (1) положительным на $\Gamma$. Согласно [5] при $q(\cdot)>0$ оператор $L u \equiv-\left(p u^{\prime}\right)^{\prime}+q u$ c условиями (2) имеет строго положительную функцию Грина, так что задача $(1),(2)$ оказывается эквивалентной задаче

$$
u(x)=\lambda \int_{\Gamma} G(x, s) r(s) u(s) d s \stackrel{\text { def }}{=} \lambda\left(G_{r} u\right)(x)
$$

с компактным и сильно положительным (в смысле естественной упорядоченности в $C(\Gamma))$ оператором $G_{r}$. Отсюда следует дискретность спектра $\Lambda$ и простота и строгая положительность минимального по модулю собственного значения $\lambda_{0}$, а также отсутствие нулей в $Г$ у соответствующей собственной функции $u_{0}(\cdot)$.

Пусть $u_{k}(\cdot)$ - одна из собственных функций задачи $(1),(2)$. Если она хотя бы на одном из ребер $\gamma_{i}$ имеет неизолированный или кратньй нуль, то $u_{k}(\cdot) \equiv 0$ на этом $\gamma_{i}$. Поэтому $u_{k}(a)=0$ на том из концов $\gamma_{i}$, который является внутренней для Г вершиной, т.е. лежит в $J(\Gamma)$. Из условия (3) тогда следует, что не менее чем для двух подграфов $\Gamma_{J_{1}}(a), \Gamma_{J_{2}}(a)$ сужение $u_{k}(\cdot)$ нетривиально, т.е. $\lambda_{k}$ принадлежит обоим спектрам $\Lambda_{j_{1}}(a), \Lambda_{j_{2}}(a)$, что противоречит предположению об общности положения. Таким образом, каждая собственная функция $u_{k}(\cdot)$ задачи $(1),(2)$ имеет в $Г$ лиш конечное число нулей и не обращается в нуль на $J(\Gamma)$; каждая из имеющихся в $\Gamma$ нулевых точек простая, т.е. $u_{k}(\cdot)$ меняет в нем знак.

Покажем теперь, что каждая точка $\lambda_{k}$ спектра $\Lambda$ является простой и строго положительной. Выбрасывая из $\Gamma$ все нули $u_{k}(\cdot)$, обозначим через $\Gamma_{0}$ любую компоненту связности оставшегося множества. Для сужения на $\Gamma_{0}$ задачи $(1),(2)$ функция $u_{k}(\cdot)$, не имеющая в $\Gamma_{0}$ нулей и равная нулю на $\partial \Gamma_{0}$, оказывается собственной функцией, являясь собственной функцией и аналогичного (4) оператора, вследствие 
чего $\lambda_{k}$ является простым и строго положительным собственным значением. Простота $\lambda_{k}$ относительно любой другой (подобной $\Gamma_{0}$ ) компоненты связности означает простоту $\lambda_{k}$ и для исходной задачи $(1),(2)$.

Покажем теперь, что $u_{k}(\cdot)$ имеет в $\Gamma$ не менее $k$ нулей. При $k=0$ это свойство очевидно. Предполагая, что $u_{k_{0}}(\cdot)$ имеет $\nu_{0}\left(>k_{0}\right)$ нулей в $\Gamma$, и выбрасывая из $\Gamma$ все эти нулевые точки $u_{k_{0}}(\cdot)$, обозначим компоненты связности оставшегося множества через $\Gamma_{0}, \Gamma_{1}, \ldots, \Gamma_{\nu_{0}}$. Так как $\lambda_{k_{0}+1}>\lambda_{k_{0}}$, то в каждом из множеств $\Gamma_{k}$ $\left(k=\overline{0, \nu_{0}}\right)$ собственная функция $u_{k_{0}+1}(\cdot)$ по теореме сравнения (теорема 8 из [5]) меняет знак, т.е. имеет в $\Gamma$ различных нулей не менее, чем $\nu_{0}+1 \geqslant k_{0}+1$. Доказательство того, что таких нулей не более $k_{0}+1$, требует значительно более тонких рассуждений (как и неограниченность $\Lambda$ ), на которых мы здесь не останавливаемся.

Институт математики

Воронежского государственного университета

Поступило 13.12 .94

\section{СПИСОК ЦИТИРОВАННОЙ ЛИТЕРАТУРЫ}

1. Nicaise S. // Lecture Notes in Math. V. 1771. N. Y.: Springer Verlag, 1985. Р. 532-541. 2. Павлов Б. С., Фадеев М. Д. // ТМФ. 1983. Т. 55. № 2. С. 257-269. 3. Пенкин О.М., Покорный Ю. В., Провоторова Е. Н. Об одной векторной краевой задаче // Краевые задачи. Пермь, 1983. С. 64-70. 4. Покорный Ю. В., Пенкин О. М. // Докл. АН СССР. 1989. Т. 309. №6. С. 1306-1309. 5. Покорный Ю. В., Пенкин О. М. // Дифференц. уравнения. 1989. Т. 25. № 7. C. $1141-1150$. 